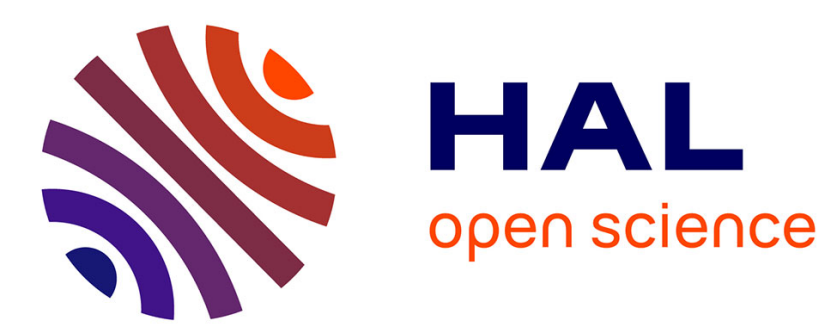

\title{
Dimer diffusion as a driving mechanism of the step bunching instability during homoepitaxial growth
}

\author{
M. Vladimirova, A. de Vita, A. Pimpinelli
}

\section{To cite this version:}

M. Vladimirova, A. de Vita, A. Pimpinelli. Dimer diffusion as a driving mechanism of the step bunching instability during homoepitaxial growth. Physical Review B: Condensed Matter and Materials Physics (1998-2015), 2001, 64, pp.245420-245426. 10.1103/PhysRevB.64.245420 . hal-00272531

\section{HAL Id: hal-00272531 \\ https://hal.science/hal-00272531}

Submitted on 11 Dec 2014

HAL is a multi-disciplinary open access archive for the deposit and dissemination of scientific research documents, whether they are published or not. The documents may come from teaching and research institutions in France or abroad, or from public or private research centers.
L'archive ouverte pluridisciplinaire HAL, est destinée au dépôt et à la diffusion de documents scientifiques de niveau recherche, publiés ou non, émanant des établissements d'enseignement et de recherche français ou étrangers, des laboratoires publics ou privés. 


\title{
Dimer diffusion as a driving mechanism of the step bunching instability during homoepitaxial growth
}

\author{
M. Vladimirova \\ Institut Romand de Recherche Numérique en Physique des Matériaux (IRRMA), PPH-Ecublens, 1015 Lausanne, Switzerland
}

\author{
A. De Vita
}

Institut Romand de Recherche Numérique en Physique des Matériaux (IRRMA), PPH-Ecublens, 1015 Lausanne, Switzerland and INFM and Dipartimento di Ingegneria dei Materiali, Università di Trieste, via A. Valerio 2, 34127 Trieste, Italy

\author{
A. Pimpinelli \\ Laboratoire des Sciences et Matériaux pour l'Electronique, et d'Automatique (LASMEA), UMR 6602 CNRS, Université Blaise Pascal, \\ Clermont 2, F-63177 Aubière Cedex, France \\ (Received 3 April 2001; revised manuscript received 5 July 2001; published 6 December 2001)
}

\begin{abstract}
The impact of ad-dimer diffusion on the morphology of an epitaxially growing stepped surface is studied analytically and by kinetic Monte Carlo simulations. It is shown that if diffusing adatoms are hindered by an Ehrlich-Schwoebel barrier at step edges, ad-dimer diffusion gives rise to a step bunching instability, provided that the corresponding Ehrlich-Schwoebel barrier for ad-dimers is small or vanishing.
\end{abstract}

DOI: 10.1103/PhysRevB.64.245420

\section{INTRODUCTION}

When growing a crystal, it is important for most applications to control the shape of the growing surface. In particular, a central problem is to understand the microscopic origin of morphological instabilities. For instance, recent experiments on semiconductor [e.g., Si (Ref. 1), GaAs (Ref. 2), InP (Ref. 3), SiC (Ref. 4)] as well as on metal vicinal surfaces [such as $\mathrm{Cu}$ (Ref. 5)] show that, depending on the growth parameters these surfaces undergo one of the two typical morphological instabilities. These are the so-called step meandering or Bales-Zangwill instability ${ }^{6}$ and the step bunching. The former type of instability is usually thought to appear on vicinal surfaces due to the Ehrlich-Schwoebel (ES) barrier, ${ }^{7,8}$ i.e., an additional energy required for a diffusing atom to step down a monatomic step. Several theoretical and experimental works have been produced with the aim of determining the ES barrier, ${ }^{9-13}$ yielding qualitatively the same result: the rate of adatom incorporation into the step is higher from the lower terrace then from the upper one. On the other hand, Schwoebel has shown that the distribution of terrace widths on a vicinal surface is stabilized during growth in the presence of an ES barrier. ${ }^{8}$ This barrier thus hinders the bunching of steps. In the same work, Schwoebel has shown that bunching may be caused by preferential incorporation of particles into steps from the upper terrace, i.e., when adatoms incorporating into the step from the lower terrace encounter an additional energy barrier. We will call this phenomenon inverse Schwoebel effect (ISE). Since step bunching during growth is frequently observed, the ISE has often been invoked as its microscopic cause. In one case, kinetic Monte Carlo (KMC) simulations based on the ISE seem to fit the experimental data well. ${ }^{14,15}$ However, justifying the ISE itself appears to be a hard task. Indeed, the ISE implies that adatoms do not easily incorporate at kinks, unless they have to step down a monatomic step to do it. This scenario seems to contradict the customary assumptions of crystal growth, which might however be too simplistic to describe recon-
PACS number(s): 68.55.-a, 81.15.Aa, 81.15.Kk

structed steps observed on semiconductor surfaces. To make things more complicated, the occurrence of step meandering and step bunching in the same system, has been reported. ${ }^{2,16}$ In such cases, one would be forced to assume that an ES barrier can coexist with an inverse Schwoebel effect, i.e., that a system may switch from ES to ISE behavior when changing the growth regime. ${ }^{2}$

Another kinetic mechanism, alternative to ISE, has recently been predicted to give rise to step bunching, namely, an enhanced adatom diffusion along step edges, with respect to surface diffusion. ${ }^{17}$ This mechanism depends on the crystallographic orientation of the steps; on a (100) surface, for instance, it should be operative for [110] steps, but not for [010] ones. ${ }^{17}$ Finally, in multicomponent systems, such as compound semiconductors, or semiconductors grown by chemical epitaxy invoking precursor molecules, the coupling between the surface densities of the diffusing species has been shown to cause step bunching, as well as step meandering, depending on the growth conditions. ${ }^{18,19}$ The goal of the present paper is to propose a microscopic, purely kinetic mechanism explaining the coexistence of step bunching and step meandering in simple systems. We stress that we address situations in which step bunching is due to deposition and growth, that is, to the surface being out of equilibrium, and does not persist if the surface is annealed. This contrasts with the step bunching obtained during annealing, whose driving force is in general the minimization of local bonding energies. ${ }^{20,21}$ Thus, we show here that the coupling between adatom and ad-dimer (or cluster) densities, which exists in single-component systems (such as $\mathrm{Cu}$ on $\mathrm{Cu}$, or $\mathrm{Si}$ on $\mathrm{Si}$ ), yields a novel kinetic pathway for the appearance of the step bunching instability during single component epitaxial growth.

The paper is organized as follows. We first introduce the step-flow growth model, which accounts for ad-dimers formation and diffusion. The model is described by a couple of nonlinear differential equations. In the second part we linearize these equations and study the stability of the step-flow growth with respect to the step bunching in the framework of the linearized model.The results are compared with the re- 
sults of the multicomponent model of Refs. 18 and 19 and discussed in terms of the surface current. In the third part, we perform one-dimensional KMC simulations of the growth process, taking into account dimer formation and diffusion. The results are then compared with the results obtained on the basis of the linearized model. Finally, the limits of the present step-flow growth model applied to systems with adparticles diffusion are discussed.

\section{STEP-FLOW MODEL FOR ADATOMS AND AD-DIMERS}

Let us consider deposition of atoms at a rate $\Phi$ on a vicinal surface of average terrace width $l$. When $l$ is small enough, or the ratio $\Phi / D_{1}$ is small enough, all atoms are captured by steps. Here

$$
D_{1}=D_{0}^{\text {at }} \exp \left[-E_{s}^{\text {at }} / k_{B} T\right]
$$

is the adatom diffusion constant, $E_{s}^{\text {at }}$ the surface diffusion activation energy for an isolated adatom, $D_{0}^{\text {at }}$ the attempt frequency, $T$ the temperature, and $k_{B}$ the Boltzmann constant. This is the classical step-flow growth model as described by Burton, Cabrera, and Frank ${ }^{22}$ (BCF). This model disregards adatom-adatom interactions; in particular, it neglects the possibility of two adatoms meeting and forming a dimer. When the temperature is reduced at fixed $\Phi$, the density of adatoms on the terraces increases, and dimers must eventually form.

If dimers are immobile, dimer formation is the starting point of island nucleation. ${ }^{23}$ However, dimers may move. ${ }^{24-29}$. Indeed, in special cases the dimers may even have a smaller diffusion energy barrier than free adatoms. ${ }^{30}$ We shall now assume that the dimers also diffuse, with a diffusion constant

$$
D_{2}=D_{0}^{\operatorname{dim}} \exp \left[-E_{s}^{\mathrm{dim}} / k_{B} T\right],
$$

$E_{s}^{\mathrm{dim}}$ and $D_{0}^{\mathrm{dim}}$ being the activation energy and the attempt frequency for dimer diffusion, respectively. When attempting to cross step edges, both adparticles experience, in general, an additional diffusion barrier, the ES barrier discussed above.

It is clear that if both adatoms and dimers experience a strong ES barrier, no step bunching is expected, since steps receive matter mostly from the terrace in front, and therefore the bunching is suppressed. ${ }^{8}$ On the other hand, by analogy with the two-species model discussed in Ref. 18, we expect that step bunching can be seen in the presence of a vanishingly small ES barrier for dimers, and of a significant ES barrier for adatoms. Thus, we assume that the ES barrier for the diffusing dimers is significantly smaller than that for the adatoms. This assumption is discussed in more detail below. Let us first investigate the implications of this assumption. The classical BCF picture can be easily modified to account for dimers. Indeed, dimer formation takes place at a rate proportional to $D_{1} c_{1}^{2}$, where $c_{1}(x)$ is the adatom density and $x$ is the coordinate orthogonal to the step direction. Calling $c_{2}(x)$ the dimer density, and performing as usual the socalled quasistatic approximation $\dot{c}_{1}=\dot{c}_{2}=0$, the various pro- cesses of deposition, adatom diffusion, and dimer formation and diffusion are described by the following two coupled nonlinear equations:

$$
\begin{gathered}
D_{1} \nabla^{2} c_{1}-2 D_{1} c_{1}^{2}+\Phi=0, \\
D_{2} \nabla^{2} c_{2}+D_{1} c_{1}^{2}=0,
\end{gathered}
$$

with appropriate boundary conditions at steps. In the extreme case of a vanishing ES barrier for dimers, we have

$$
\begin{aligned}
& \left.c_{1}\right|_{x=-l / 2}=0, \\
& \left.c_{2}\right|_{x=-l / 2}=0
\end{aligned}
$$

at the ascending step and

$$
\begin{gathered}
\left.D_{1} \nabla c_{1}\right|_{x=l / 2}=-\left.\nu c_{1}\right|_{x=l / 2}, \\
\left.c_{2}\right|_{x=l / 2}=0
\end{gathered}
$$

at the descending step edge. These boundary conditions account for a nonvanishing ES barrier for adatoms $E_{\mathrm{ES}}$ through the kinetic parameter $\nu$ in Eq. (5), $\nu$ being defined as $\nu$ $=D_{0} \exp \left[-\left(E_{s}+E_{\mathrm{ES}}\right) / k_{B} T\right]$. Contrary to the classical BCF model, Eqs. (3) with the boundary conditions given by Eqs. (4) and (5), are nonlinear, and an analytic solution cannot be found. We have then to resort to a numerical treatment. However, we can get a feeling of the exact behavior if we first linearize Eqs. (3) and solve the linearized equations analytically. We will see shortly that the linearized model is equivalent to the two-particle model of Ref. 18, so that the conclusions of that work qualitatively apply to the present case.

\section{LINEARIZED MODEL}

To linearize Eqs. (3) we write the square $c_{1}^{2}(x)$ as $\bar{c}_{1} c_{1}(x)$ and look for the solution $c_{1}(x)$ of the linear system

$$
\begin{gathered}
D_{1} \nabla^{2} c_{1}-2 D_{1} \bar{c}_{1} c_{1}+\Phi=0, \\
D_{2} \nabla^{2} c_{2}+D_{1} \bar{c}_{1} c_{1}=0,
\end{gathered}
$$

where the "average adatom density" $\bar{c}_{1}$ is defined selfconsistently as

$$
\bar{c}_{1}=\frac{1}{l} \int_{-l / 2}^{l / 2} c_{1}\left(x ; \bar{c}_{1}\right) d x .
$$

In Eqs. (6), $2 D_{1} \bar{c}_{1}$ has the meaning of the inverse lifetime of an adatom before dimer formation, or equivalently, $\sqrt{1 /\left(2 \bar{c}_{1}\right)}$ plays the role of a diffusion length $\lambda$ for the adatoms. On the other hand, when adatoms disappear dimers are produced at a rate $D_{1} \bar{c}_{1} c_{1}$.

Of course, this linearization scheme is rather arbitrary. Its main usefulness consists of showing that step bunching is in principle possible through the "dimer diffusion" mechanism. Our choice is motivated by the formal analogy with Ref. 18 - the roles of "precursors" and "growth units" of Ref. 18 being played here by adatoms and dimers, respectively. Indeed, the analogy requires that the dimer production rate and 


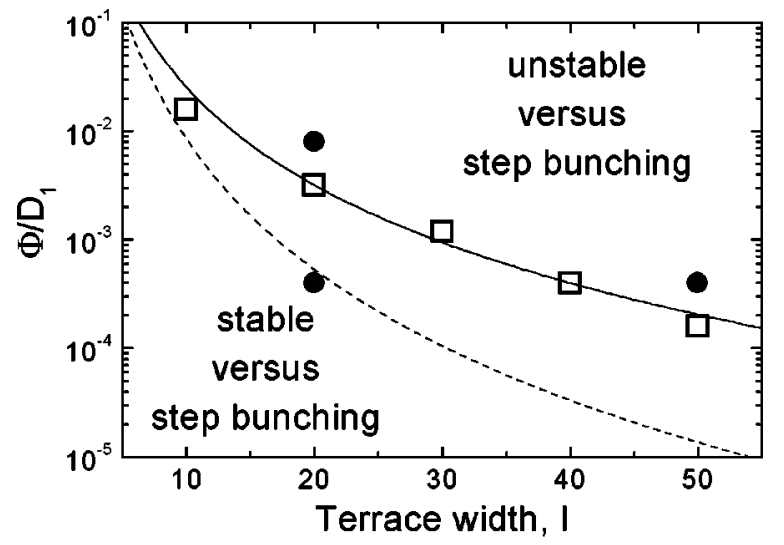

FIG. 1. Kinetic stability diagram at $723 \mathrm{~K}$ obtained from the linearization procedure (solid and dashed lines for 1D and 2D surfaces, respectively), and from 1D KMC simulations (open squares). Solid circles indicate the growth parameters corresponding to the simulated surface profiles shown in Fig. 2.

the adatom vanishing rate concide. This is true if in Eqs. (6) the second equation is multiplied throughout by 2 . Then, as anticipated, Eq. (6) formally coincides with the differential equations describing a two-component system in Ref. 18, provided the diffusion constant of the growth units, $D_{A}$, is set equal to twice the diffusion constant of the dimers, $D_{A}$ $=2 D_{2}$. Since the result of the linear stability analysis performed in Ref. 18 is independent of $D_{A}$, it applies completely to the present situation. We conclude that a vicinal surface growing by step flow is unstable with respect to step bunching if the adatom diffusion length $\lambda$ satisfies

$$
\lambda>1.51 l,
$$

provided that the ES barrier is infinite for the adatoms and vanishing for the dimers. A schematic kinetic phase diagram in the dimensionless parameter space $\left(\Phi / D_{1}, l\right)$ is shown in Fig. 1. The boundary resulting from the condition $\lambda=1.51 \mathrm{l}$ is drawn as a dashed line. It separates the region of linearly stable step-flow (lower region) from the region of linearly unstable step-flow (upper region), with respect to the step bunching instability. To obtain this result we set the temperature to $T=723 \mathrm{~K}$ and the hopping rates and diffusion barriers to $D_{0}^{\mathrm{at}}=D_{0}^{\mathrm{dim}}=10^{13} \mathrm{~s}^{-1}$ and $E_{S}^{\mathrm{at}}=E_{S}^{\mathrm{dim}}=1 \mathrm{eV}$, respectively. We then vary the incident flux $\Phi$ for each value of average terrace width $l$.

It is essential to note that relevant parameters controlling the surface stability versus step bunching are the ratio $\Phi / D_{1}$ and $l$. This is due to the fact that only the adatom (not addimer) diffusion constant matters in determining stability/ instability of step flow. Indeed, it is easy to show that $\bar{c}_{1}$ $\approx \Phi l^{2} / D_{1}$, so that the stability boundary can be written as $\lambda \approx l_{c} \approx\left(D_{1} / \Phi l^{2}\right)^{1 / 2}$, or

$$
\Phi / D_{1} \approx l_{c}^{-4} .
$$

Owing to this scaling form, the location of the boundary in the $\left(\Phi / D_{1}, l\right)$ parameter space does not depend on the temperature and can be computed numerically using a fixed temperature.
To get a qualitative argument explaining the dimer induced instability, one should bear in mind that the stability criterion given by Eq. (8) is obtained by looking at the sign of the derivative of the net average diffusion current of adparticles on the surface, as a function of the average terrace width. ${ }^{35}$ The average current is defined as the difference between the number of particles that are incorporated into the descending step, and those that are incorporated into the ascending one. Then, a positive value is assigned to the current flowing in the downhill direction-that is, more atoms incorporating into the descending step than into the ascending one. The net surface current vanishes in the absence of barriers since in this case as many particles are incorporated into the ascending step as into the descending one. Let us determine the behavior of the current qualitatively. Consider a terrace whose width is smaller than the single-atom diffusion length $\lambda$. Only few dimers form, and most diffusing particles are adatoms that experience an ES barrier at the descending step and incorporate at the ascending step. This yields a nonvanishing negative (uphill) current, which decreases (becomes more negative) as the terrace width initially increases. If a terrace is much wider than $\lambda$, the situation is more complicated. The adatoms on the terrace may have three different fates:

(i) Adatoms falling within a distance $\lambda$ from an ascending step are incorporated there. The adatom density in this region may be estimated as $c_{1} \approx \Phi \lambda^{2} / D_{1}$, which yields a current $J$ $\approx-D_{1} c_{1} / \lambda \approx-\Phi \lambda$.

(ii) Adatoms falling near the center of the terrace transform into dimers and diffuse half to the ascending and half to the descending step. They do not contribute to the net current.

(iii) Adatoms falling within a distance $\lambda$ from the descending step try to step down, but are repelled by the ES barrier. However, since $l \gg \lambda$, they are more likely to try to step down again instead of diffusing to the ascending step. Indeed, the likelihood of a random walker to hop over a distance $l$ in a given direction being proportional to $1 / l$, only a fraction $1 / l$ of such adatoms is expected to reach the ascending step. The rest eventually transform into dimers and step down, so that they approximately cancel the current of the adatoms from the lower terrace, leaving only a contribution $-\Phi \lambda / l$ to the net current.

Summarizing, the adparticle current on a terrace is always negative for any terrace width, but it vanishes as $-1 / l$ at large $l$, which implies an instability versus step bunching. ${ }^{23}$ Moreover, in agreement with the analytic results of Ref. 23, the stability boundary is determined by the relation between $\lambda$ and $l$ only. As such, it is independent of the value of the diffusion constant of the dimers, provided the latter does not vanish altogether, again in agreement with the analytic result [cf. Eq. (9)]. To complete the discussion, we tested by KMC simulations that the instability, if it appears when $D_{2}=D_{1}$, is not suppressed by increasing or decreasing $D_{2}$ by a factor of 10. The same variation of $D_{2}$ does not make the instability appear, if it is absent for $D_{2}=D_{1}$. However, the dimer diffusion constant does matter when island formation on the 
terraces is concerned. Step flow is then favored by fast dimer diffusion, and step flow is an obvious prerequisite for step bunching.

\section{KMC SIMULATIONS}

We turn now to the kinetic Monte Carlo simulations of the growth process. We use the standard KMC algorithm as described by Kellogg. ${ }^{31}$ Atoms are deposited on a onedimensional (1D) lattice with different deposition rates $\Phi$. This lattice represents a vicinal surface with average terrace width $l{ }^{32}$ Adatoms then perform a random walk with hopping rate $D_{1}$. They are either incorporated at steps or meet pairwise to form dimers. Once formed, the dimers also diffuse at a rate $D_{2}$ until they are incorporated at a step edge. The values of diffusion parameters and the temperature are chosen as reported in Sec. III. The incorporation at steps of both adparticles is assumed to be irreversible. The dissociation of dimers on the terrace is forbidden as well as the formation of clusters larger than dimers. Thus, the validity of the model is restricted to the step-flow growth regime. The presence of the ES barrier at upper step edges is accounted for by lowering the probability of hopping down a step by a factor $\exp \left(-E_{\mathrm{ES}} / k_{B} T\right)$, and we start with the most favorable case: an infinite ES barrier for adatoms $\left(E_{\mathrm{ES}}^{\mathrm{at}}=1 \mathrm{eV}\right)$ and a vanishing barrier for dimers $\left(E_{\mathrm{ES}}^{\mathrm{dim}}=0\right)$. In order to locate the boundary between stable step flow and step bunching, we proceed in the following way: for each vicinal surface with a given average terrace width $l$ we start with a high deposition rate, where step bunching is seen after depositing 5000 monolayers (ML). We then progressively lower the deposition rate in steps of one unit in a logarithmic scale (i.e., from 400 to $300 \mathrm{ML} / \mathrm{s}$, or from 20 to $10 \mathrm{ML} / \mathrm{s}$, and so on) until step bunching is lost. In the phase diagram of Fig. 1 we report the smallest tested value of the deposition rate where bunching is observed (open squares).

Figure 2 illustrates the surface profiles, resulting from stable (a) and unstable $(\mathrm{b}, \mathrm{c})$ growth regimes. In the parameter space $\left(l, \Phi / D_{1}\right)$ (Fig. 1$)$ these regimes are represented by filled circles. When the average step distance is $l=20$ and $\Phi / D_{1}=4 \times 10^{-4}$ [Fig. 2(a)], which falls in the stable stepflow region, the step train remains rather uniform even after deposition of 5000 ML. The correspondent terrace width distribution (TWD) that gives the probability of finding a terrace of a given width on the surface [Fig. 2(a), inset] can be fitted with a Gaussian centered at $l_{0}=20$ with standard deviation $\sigma=5$. Keeping $l=20$ but increasing the deposition rate so that $\Phi / D_{1}=8 \times 10^{-4}$ puts the system in the unstable region. The resulting surface morphology is characterized by the coexistence of high and low step density regions. The bimodal character of the corresponding TWD [Fig. 2(b), inset], is a signature of the step bunching instability. ${ }^{33}$ Indeed, this TWD can be fitted with the weighted sum of two Gaussians. These curves are shown in the inset of Fig. 2(b) by solid lines. The fitting parameter values are as follows: mean value $l_{0}=12$ (26), standard deviation $\sigma=16$ (8) and area $a=0.4$ (0.6) for Gaussians describing short (large) terrace width distribution. Finally, step bunching also appears when the ratio $\Phi / D_{1}$ is set to $4 \times 10^{-4}$ as in Fig. 2(a), and $l$ to 50
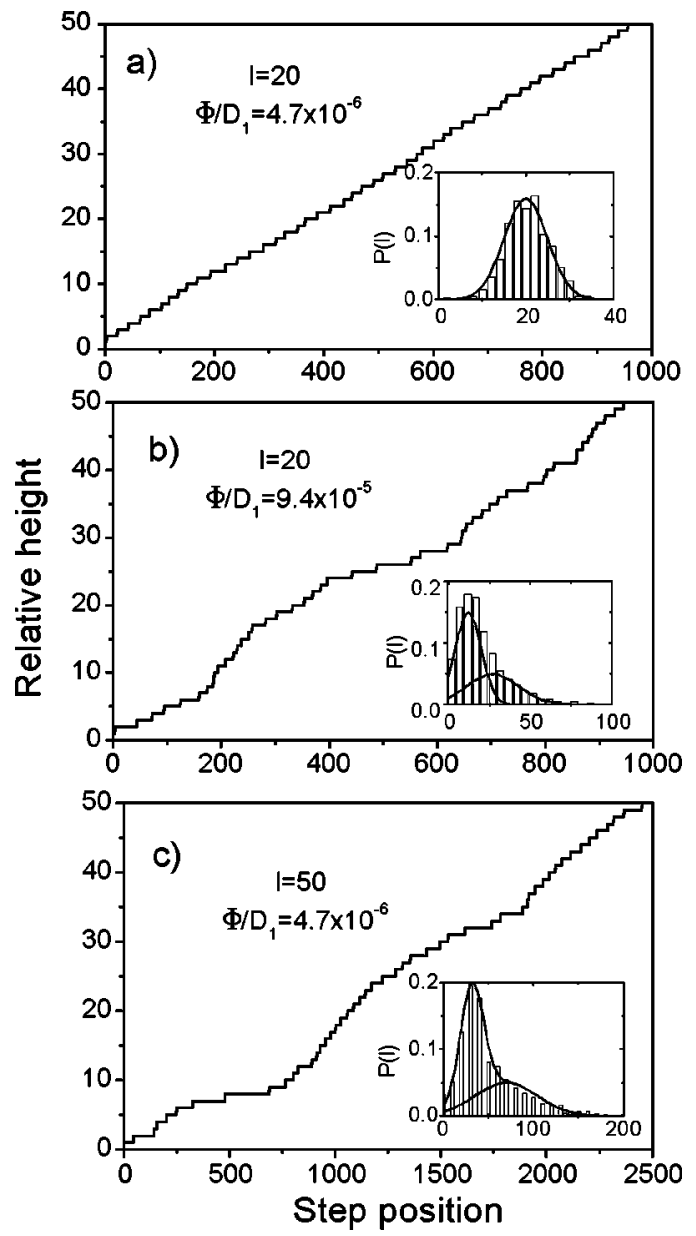

FIG. 2. Surface profiles resulting from KMC simulations after deposition of $5000 \mathrm{ML}$ at $723 \mathrm{~K}, E_{\mathrm{ES}}^{\mathrm{at}}=1 \mathrm{eV}, D_{0}^{\mathrm{at}}=D_{0}^{\mathrm{dim}}$ $=10^{13} \mathrm{~s}^{-1}, E_{S}^{\mathrm{at}}=E_{S}^{\mathrm{dim}}=1 \mathrm{eV}$. The average terrace width $l=20$ $(\mathrm{a}, \mathrm{b})$ and $l=50(\mathrm{c})$, the ratio between the deposition and adatom diffusion rates is $\Phi / D_{1}=4 \times 10^{-4}$ (a,c) and $\Phi / D_{1}=8 \times 10^{-4}(\mathrm{~b})$. The insets show the TWD on the surface together with its fit by a single (a) or double $(\mathrm{b}, \mathrm{c})$ Gaussian distribution. The corresponding points of the $\left(l, \Phi / D_{1}\right)$ parameter space are marked by solid circles in Fig. 1.

[Fig. 2(c)]. Again, the TWD [Fig. 2(c), inset] exhibits two components. Fitting with the sum of two Gaussians gives mean value $l_{0}=32$ (72), standard deviation $\sigma=13$ (35), and area $a=0.56(0.44)$ for short (large) terrace distribution.

Finally, we compare the simulations with the analytic results. A word of caution is needed at this point. The instability boundary (9) (dashed line in Fig. 1) is obtained for a two-dimensional (2D) surface, while the simulations are performed on a 1D lattice. On the other hand, it is known that the adatom pairing rate depends on the surface dimension. ${ }^{34}$ One can show that the diffusion length of adatoms before dimer formation is $\lambda=1 / \bar{c}_{1}^{2}$ in two dimensions, while $\lambda$ $=1 / \bar{c}_{1}$ in the $1 \mathrm{D}$ case. Thus, from the criterion (8) we obtain the scaling relation for the instability boundary in the 1D case:

$$
\Phi / D_{1} \approx l_{c}^{-3} .
$$


This relation is shown in Fig. 1 as a solid line and describes rather well the instability boundary obtained from 1D KMC simulations.

\section{DISCUSSION AND SUMMARY}

The results described in the previous sections show that the proposed adatom-dimer mechanism is indeed able to make a vicinal surface unstable with respect to step bunching, when it is growing in a step-flow mode. However, to obtain a realistic model, four main elements should be added: (i) the 2D character of a real surface, (ii) the possibility of island nucleation on the terraces between steps, (iii) dimer dissociation on the open terraces, and (iv) physically relevant ES barriers for adparticles.

The first point is not expected to introduce any qualitative change. The characteristics of step bunching in the present model are essentially the same as in the fully 2D model studied in Ref. 19. In particular, a clear "phase separation" between a step-rich and a step-poor region is seen in 1D as well as $2 \mathrm{D}$ space.

Accounting for island nucleation (ii) is a more delicate point. It is clear that island nucleation is favored when the ratio $\Phi / D_{1}$ is large, and/or the terraces are wide-which is just what bunching requires to be seen. Therefore, at very high deposition rates, or very high temperatures, growth by island formation ultimately takes over the step-flow regime, the BCF approach loses its validity, and Eqs. (3) no longer describe the growing surface.

However, the threshold for island nucleation is a materialdependent property. It will depend on the surface symmetry, and, especially for semiconductors, on the presence of reconstruction-driven anisotropies of sticking, diffusion, etc. It will also depend, of course, on the presence of strongly diffusing dimers, or larger clusters. ${ }^{36}$ As we said in the Introduction, too little is known about dimer and cluster diffusion to be of help. To understand the difficulty, consider that in order to make an estimate one should be able to compare the scaling relation for the instability boundary, $l_{c} \approx F^{-1 / 4}$, with the scaling relation linking the average distance between islands, $l_{s}$, with the deposition rate. The latter is known in a few cases: it is of the form $l_{s} \approx F^{-2 / 5}$ when dimer diffusion is isotropic, ${ }^{34}$ and of the form $l_{s} \approx F^{-1 / 4}$ when clusters of all sizes diffuse. ${ }^{37}$ In both cases, whether islands nucleate before the steps bunch or not depends on the prefactors, and those in turn depend on the specific material and are in general not known.

Another effect of increasing the temperature is the dissociation of dimers (iii). Therefore, it is necessary to verify that the step bunching instability persists in case when dimer formation is reversible, that is, when dimers break up into adatoms after a certain time, on average. We can roughly estimate that the instability induced by the diffusion of stable dimers is not destroyed by the dissociation if the dimer diffusion length exceeds the average terrace width. Thus, denoting the dimer binding energy as $E_{b}$ so that the dimer diffusion length is $\lambda^{\operatorname{dim}} \approx \exp \left(E_{b} / k_{B} T\right)$ we obtain the condition $E_{b}>k_{B} T \ln (l)$ on the dimer binding energy. If this condition is satisfied, the mechanism proposed in the present work be- comes operative. Using KMC simulations we check this condition in the particular case of the growth regime illustrated in Fig. 2(b) $(l=20, T=723 \mathrm{~K})$. The step bunching appears if $E_{b}$ is set to $0.2 \mathrm{eV}$, while the surface remains stable if $E_{b}=0.1 \mathrm{eV}$, in agreement with our estimation.

Finally we discuss the choice of $E_{\mathrm{ES}}$ for the adparticles (iv). In Ref. 18 the linear stability analysis is performed also in the case of more general boundary conditions for the two types of particles: (a) finite Schwoebel effect for the adatoms and vanishing one for the dimers; (b) infinite Schwoebel effect for the adatoms and finite one for the dimers. In both cases it is shown that the bunching instability persists for physically relevant values of the barriers. In particular, it is always found in case (a), while it is found in case (b) only for not-too-high ES barriers for the dimers. In fact, it is clear that in the presence of high barriers for both adatoms and dimers, the present model would be the analog of a one-particle model with ES barriers, which does not exhibit step bunching. A useful criterion for deciding whether a given ES barrier is high or not (equivalently, whether a given ES effect is strong or not) is to compute the length $l_{\mathrm{ES}}=\exp \left(E_{\mathrm{ES}} / k_{B} T\right)$, and to compare it with the terrace width $l$. A strong ES effect (a high barrier) is implied by $l_{\mathrm{ES}} \gg l$, and vice versa. For example, using our criterion for $l=50$ and $T=723 \mathrm{~K}$, we find that a barrier is high if it is bigger than $0.25 \mathrm{eV}$, and low otherwise. We have checked this criterion numerically. Once the parameter set is chosen in the unstable region of Fig. 1, steps eventually bunch for any finite ES barrier for the adatoms and a vanishing barrier for dimers. Choosing $E_{\mathrm{ES}}$ $=0.5 \mathrm{eV}$ for the adatoms (a high but not unphysical value), bunching appears when the dimers' ES barrier is $0.1 \mathrm{eV}$ and does not appear when the barrier is $0.3 \mathrm{eV}$, in agreement with our estimation.

In conclusion, we have proposed a mechanism that may be responsible for the step bunching instability observed during elemental homoepitaxial growth on vicinal surfaces. The main role is played by diffusing dimers, which are assumed to experience a small ES barrier when crossing steps, whereas diffusing adatoms experience a strong ES barrier at step edges. This assumption is, e.g., justified for the $\mathrm{Si}(001)$ surface, where the absence of the ES barrier for dimers is predicted. ${ }^{10}$ In the framework of the present model we can distinguish three growth regimes. The step-flow growth (i) takes place when the adatoms are incorporated at step edges before forming dimers or nucleating islands. If this is the case, our model is just a one-particle model in the presence of an ES barrier at the step edge. As shown by Schwoebel, ${ }^{8}$ this implies that a vicinal surface is stable against step bunching and unstable against step meandering. Step bunching appears in the intermediate regime (ii) when the step advancement is mainly due to the incorporation of diffusing dimers. At higher deposition rates (depending on the terrace width) growth by island formation eventually takes over (iii), and the model behaves again as a standard one-particle system with a step-edge barrier. Thus, combining analytic calculations in the framework of the BCF model with $\mathrm{KMC}$ simulations, we have shown that the diffusion of dimers on the vicinal surface may lead to step bunching, when the average terrace width is larger than a critical value that depends on the deposition rate and the growth temperature. 
${ }^{1}$ A.L. Aseev, A.V. Latyshev, and A.B. Krasilnikov, Surf. Rev. Lett. 4, 551 (1997); Jian-hong Zhu, K. Brunner, and G. Abstreiter, Appl. Phys. Lett. 73, 2438 (1998).

${ }^{2}$ P. Tejedor, F.E. Allegretti, P. Šmilauer, and B.A. Joyce, Surf. Sci. 407, 82 (1998); P. Tejedor, P. Šmilauer, C. Roberts and B.A. Joyce, Phys. Rev. B 59, 2341 (1999); P. Tejedor, P. Šmilauer, and B.A. Joyce, Surf. Sci. 424, L309 (1999).

${ }^{3}$ H. Dumont, Y. Monteil, and J. Bouix, Appl. Surf. Sci. 161, 286 (2000).

${ }^{4}$ M. Syvajarvi, R. Yakimova, T. Iakimov, and E. Janzen, Mater. Sci. Forum 338-342, 375 (2000).

${ }^{5}$ J. Camarero et al., Appl. Phys. A: Mater. Sci. Process. 69, 553 (1999).

${ }^{6}$ G.S. Bales and A. Zangwill, Phys. Rev. B 41, 5500 (1990).

${ }^{7}$ G. Ehrlich and F.G. Hudda, J. Chem. Phys. 44, 1039 (1966).

${ }^{8}$ R.L. Schwoebel, J. Appl. Phys. 40, 614 (1968).

${ }^{9}$ F. Family and J. G. Amar, in Proceedings of the 1995 Simulation Multiconference, edited by A. Tentner (The Society for Computer Simulation International, San Diego, CA, 1995), p. 234.

${ }^{10}$ E. Kim, C.W. Oh, and Y.H. Lee, Phys. Rev. Lett. 79, 4621 (1997).

${ }^{11}$ P.J. Feibelman, Phys. Rev. Lett. 81, 168 (1998).

${ }^{12}$ G.S. Icking-Konert, M. Giesen, and H. Ibach, Surf. Sci. 398, 37 (1998).

${ }^{13}$ K. Morgenstern, G. Rosenfeld, E. Laegsgaard, F. Besenbacher, and G. Comsa, Phys. Rev. Lett. 80, 556 (1998).

${ }^{14}$ T. Fukui and H. Saito, Jpn. J. Appl. Phys., Part 2 29, L483 (1990); T. Fukui, J. Ishizaki, S. Hara, J. Motohisa, and H. Hasegawa, J. Cryst. Growth 146, 183 (1995); J. Ishizaki, K. Ohkuri, and T. Fukui, Jpn. J. Appl. Phys., Part 1 35, 1280 (1996); Y. Oda and T. Fukui, J. Cryst. Growth 195, 6 (1998); M. Akabori, J. Motohisa, and T. Fukui, ibid. 195, 579 (1998).

${ }^{15}$ M. Krishnamurthy, M. Wassermeier, D.R.M. Williams, and P.M. Petroff, Appl. Phys. Lett. 62, 1922 (1993).

${ }^{16} \mathrm{~J}$. Ernst (private communication).

${ }^{17}$ P. Politi and J. Krug, Surf. Sci. 446, 89 (2000).
${ }^{18}$ A. Pimpinelli and A. Videcoq, Surf. Sci. Lett. 445, L23 (2000).

${ }^{19}$ M. Vladimirova, A. Pimpinelli, and A. Videcoq, J. Cryst. Growth 200, 631 (2000).

${ }^{20}$ S.V. Khare, T.L. Einstein, and N.C. Bartelt, Surf. Sci. 339, 353 (1995).

${ }^{21}$ M.I. Larsson, Phys. Rev. B 56, 15157 (1997).

${ }^{22}$ W.K. Burton, N. Cabrera and F. Frank, Philos. Trans. R. Soc. London, Ser. A 243, 299 (1951).

${ }^{23}$ A. Pimpinelli and J. Villain, Physics of Crystal Growth (Cambridge University Press, Cambridge, 1998).

${ }^{24}$ K. Kyuno, A. Golzhauser, and G. Ehrlich, Surf. Sci. 397, 191 (1998).

${ }^{25}$ M. Krueger, B. Borovsky, and E. Ganz, Surf. Sci. 385, 146 (1997).

${ }^{26}$ D.G. Papageorgiou, and G.A. Evangelakis, Surf. Sci. 461, L543 (2000).

${ }^{27}$ C.M. Chang, C.M. Wei, and S.P. Chen, Phys. Rev. Lett. 85, 1044 (2000).

${ }^{28}$ G.D. Lee, C.Z. Wang, Z.Y. Lu, K.M. Ho, Surf. Sci. 426, L427 (1999).

${ }^{29}$ A. Bogicevic, S. Ovesson, B.I. Lundquist, and D.R. Jennison Phys. Rev. B 61, R2456 (2000).

${ }^{30}$ P.J. Feibelman, Phys. Rev. B 61, R2452 (2000).

${ }^{31}$ G.L. Kellogg, Surf. Sci. Rep. 21, 1 (1994).

${ }^{32}$ We adjust the total length $L$ of the one-dimensional lattice so that $L=l n_{\text {step }}$, where the terrace width $l$ is varied and the number of steps on the lattice $n_{\text {step }}$ is set to 50 in all cases.

${ }^{33}$ A. Videcoq, A. Pimpinelli, and M. Vladimirova, Appl. Surf. Sci. , 177, 213 (2001)

${ }^{34}$ A. Pimpinelli, J. Villain, and D.E. Wolf, Phys. Rev. Lett. 69, 985 (1992).

${ }^{35}$ J. Villain, J. Phys. I 1, 19 (1991).

${ }^{36}$ S. Liu, L. Bönig, and H. Metiu, Phys. Rev. B 52, 2907 (1995).

${ }^{37}$ P.L. Krapivsky, J.F.F. Mendes, and S. Redner, Phys. Rev. B 59, 15950 (1999). 